\title{
Estrategia didÁctica PARA LA ENSEÑANZA dE LA QuímicA EN el contexto de relaciones Ciencia, Tecnología, Sociedad y Ambiente
}

\author{
DidActic eStRATEGY TO TEACH CHEMISTRY IN THE CONTEX OF \\ relations of Science, Technology, Society and Environment
}

\author{
Jaime Enrique Guerrero Santafé* \\ jaiguer@uni.pedagogica.edu.co \\ Luz Marina Cabrera Morillo** \\ lcabrera@udca.edu.co
}

\section{Resumen}

El propósito de la presente publicación es discutir y analizar la propuesta interdisciplinar para la enseñanza de la química, utilizando como objetos de trabajo las actividades humanas desde la perspectiva de las relaciones ciencia, tecnología, sociedad y ambiente. Las acciones metodológicas se refieren a procesos de conceptualización, experimentación, problemas de investigación y estrategias de manejo ambiental.

Palabras clave: Combustión, Oxido-Reducción, Dureza del Agua, Alcalinidad, Gases Invernadero, Biomasa, Energía Renovable, Reacciones Anaeróbicas, Enzimas, Combustibles Fósiles, Calentamiento Global, Lluvia ácida, Lluvia Seca, Smog Fotoquímico.

\section{Abstract}

The purpose of this publication is to discuss and analyze the interdisciplinary proposal for the teaching of Chemistry using human activities as objects of study in the perspective of Science, Technology, Society and Environment. The methodological actions are related to conceptualization, experimentation, research problems, environmental strategies and management.

\footnotetext{
* Profesor Titular Universidad Pedagógica Nacional

**. Universidad de Ciencias Aplicadas y Ambientales
} 
Key words: Didactic Strategy, Science, Technology, Society and Environment, Oxidation-reduction, Global warning, Biomass, Renovable Energy, Fosil fuels, Acid rain, Anaerobic Bacteria, Fotochemical Smog, Hard Water, Alkalinity.

\section{INTRODUCCIÓN}

Las interacciones humanas con los componentes del ambiente han procurado sustento y beneficio para las comunidades en el transcurso de su evolución histórica. En el comienzo de las civilizaciones humanas, dado el escaso número de personas y su percepción del entorno, estas interacciones no produjeron impactos ambientales significativos. Los avances científicos y tecnológicos aportaron herramientas y conocimiento para obtener mayor beneficio del entorno; lo cual permitió el crecimiento de las poblaciones y la explotación de nuevos materiales, que originaron la acumulación e intercambio de productos y la aparición de notorias alteraciones ambientales.

Las sociedades modernas no sólo han considerado el ambiente como un soporte para su sustentabilidad, sino también como un medio de beneficio económico creciente que utiliza grandes cantidades de materiales y de energía, y pone en riesgo la sostenibilidad del planeta.

En este ámbito, la estrategia interdisciplinar que se propone aborda un proceso didáctico para la enseñanza de la Química teniendo en cuenta las actividades humanas y los procesos químicos como objetos de estudio o problemas de trabajo en el contexto de ciencia, tecnología, sociedad, ambiente, propiciando el desarrollo de competencias individuales para la comprensión e interrelación de estas complejas interacciones mediante actividades de conceptualización, experimentación, y estrategias de manejo de la problemática.

\section{Estrategia Didáctica para la enseñanza de la Química}

La estrategia didáctica que utiliza las actividades humanas como objeto de trabajo establece una profunda relación con los conceptos de la ciencia, en especial con los de la química. Cualquier actividad humana tie- ne lugar en un entorno específico y está relacionada con hechos científicos, tecnológicos, sociales y ambientales. El estudio de estas relaciones facilita la construcción de competencias necesarias para compren- 
der las complejas situaciones ambientales resultantes y asumir con responsabilidad y compromiso las acciones llevadas a cabo en el ambiente.

Las etapas de la estrategia didáctica incluyen la selección de una actividad humana; la identificación de los conceptos clave de la química involucrados, su descripción, el establecimiento de relaciones entre los conceptos; la experimentación; los problemas de investigación; las estrategias de manejo y las implicaciones en el contexto de la ciencia, la tecnología, la sociedad y el ambiente. A continuación se da un ejemplo de la estrategia para la actividad de combustión.

Actividad Humana Uso de gas metano como combustible en la obtención de energía para diversos propósitos.

\section{Conceptos Clave} Combustión, Oxido-Reducción, Dureza del Agua, Alcalinidad, Gases Invernadero, Fotosíntesis, Biomasa, Energía Renovable, Reacciones Anaeróbicas, Enzimas, Combustibles Fósiles, Calentamiento Global, Lluvia ácida, Lluvia Seca, Smog Fotoquímico.

DESCRIPCIÓN DE CONCEPIOS Oxidación-Reducción

La definición más importante sobre ácidos y bases, dada por Gilbert Newton Lewis, se refiere al ácido como aceptor de electrones y a la base como dador de electrones. Los agentes reductores se consideran dadores de electrones y los agentes oxidantes como aceptores de electrones.
Es importante distinguir entre reacciones ácido-base y reacciones Redox, porque la terminología es similar.

1. La característica que destaca una reacción ácido - base, es que ningún átomo cambia su estado de oxidación formal.

2. Una reacción Redox también puede ocurrir entre un dador de electrones y un aceptor de electrones, pero la trasferencia de electrones hace que un átomo incremente su estado de oxidación y el otro átomo disminuya sus estados de oxidación.

3. La transferencia de átomos se da con frecuencia, pero al mismo tiempo el átomo continúa llevando sus electrones de enlace con él.

$$
\mathrm{X}-\mathrm{O}+\mathrm{Y} \longrightarrow \mathrm{X}+\mathrm{O}-\mathrm{Y}
$$

Esta no es una característica de la reacciones Redox por cuanto la transferencia de electrones es el aspecto clave.

1. Una base dona sus electrones, pero retiene parcialmente la "propiedad" de ellos en los orbitales de valencia, mientras que el agente reductor transfiere sus electrones por completo, aunque haya o no transferencia del átomo.

2. En un sentido mecanicista, una reacción de transferencia electrónica casi siempre ocurre como una secuencia de dos etapas: 
transferencia de un electrón o transferencia de dos electrones, sin tener en cuenta el número total de electrones transferidos en la reacción estequiométrica.

3. La etapa de transferencia de un electrón por rareza involucra transferencia del átomo y de los cambios principales en la esteoquiometría de las especies reaccionantes.

4. Las etapas de transferencia de dos electrones proceden, con frecuencia por transferencia del átomo y casi siempre indican cambios mayores en el número de coordinación o al menos en la geometría molecular del dador y aceptor.

\section{Bases moleculares para transfrencia de electrones}

Las reacciones Redox por lo general están favorecidas entálpicamente y no entrópicamente.

La transferencia de electrones produce un ordenamiento electrónico general energéticamente más favorable. Las fuentes de cambios entálpicos favorables en reacciones ácido-base nos llevan a investigar las fuentes de los cambios entálpicos Redox.

Existen dos mecanismos diferentes de transferencia de electrones por cuanto las fuentes principales de energía son también diferentes para los dos: Mecanismos de esfera-interior y Mecanismos de esfera-exterior.

1. En el mecanismo de esfera interior, el átomo oxidado y el átomo reducido forman enlaces dirigidos a un átomo común o grupo molecular pequeño, el cual sirve luego como puente para la transferencia del electrón.

$$
\mathrm{AX}+\mathrm{B}\left(\mathrm{H}_{2} \mathrm{O}\right) \rightarrow \mathrm{AXB}+\mathrm{H}_{2} \mathrm{O} \text { (en agua) }
$$

$\mathrm{AXB} \rightleftarrows \mathrm{AXB}^{*}$ (complejo activado)

$\mathrm{AXB}^{*} \rightleftarrows \mathrm{A}-\mathrm{X}-\mathrm{B}^{+}$(átomo transferido)

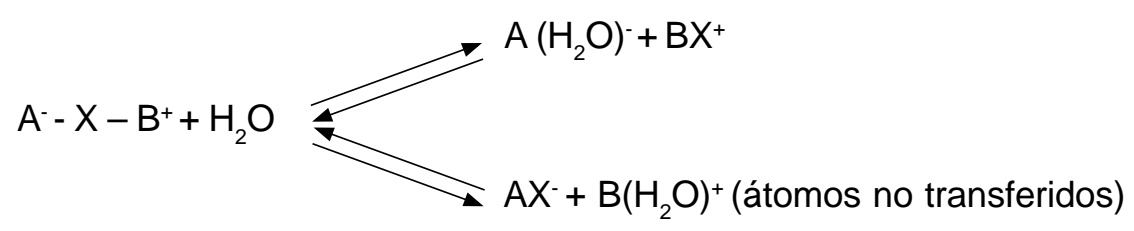


El grupo puente no se oxida ni se reduce. Átomos de oxígeno y de halógenos son átomos puente comunes, pero algunos grupos moleculares pequeños como el ión cianuro $\mathrm{CN}^{-}$pueden cumplir esta función.

2) En el mecanismo de esfera exterior, el átomo que se oxida y el átomo que se reduce pueden encontrarse directamente (reacción sodio metálico con cloro gaseoso).
En el caso de una solución, se encuentra que sus esferas de solvatación y esferas de coordinación se tocan, sin la formación de ningún nuevo enlace dirigido. Si utilizamos la notación // (paralelo) para representar contacto entre electrones de los niveles de valencia de los dos átomos sin compartir electrones, o la formación de enlaces, el mecanismo procedería así:

$$
\begin{aligned}
& \mathrm{A}+\mathrm{B} \longrightarrow \mathrm{A}^{/ / \mathrm{B}} \\
& \mathrm{A} / / \mathrm{B} \longrightarrow \mathrm{A}^{/ / \mathrm{B}^{*}} \\
& {\mathrm{~A} / / \mathrm{B}^{*}} \rightleftarrows \mathrm{A}^{-} / / \mathrm{B}^{+} \\
& \mathrm{A}^{-} / / \mathrm{B}^{+}
\end{aligned}
$$

Esta notación es apropiada en el caso que A y B sean átomos individuales o especies moleculares coordinadas. Consideremos la fuerza que favorece termodinámicamente la reacción de esfera exterior.

$$
\mathrm{Na}_{(\mathrm{s})}+1 / 2 \mathrm{Cl}_{2(\mathrm{~g})} \longrightarrow \mathrm{NaCl}_{(\mathrm{s})}
$$

Para esta reacción muy espontánea se tienen los siguientes datos termodinámicos:

$\Delta \mathrm{G}^{0}=-91.8 \mathrm{Kcal} / \mathrm{mol}$ (espontánea)

$\Delta \mathrm{H}^{0}=-98.2 \mathrm{Kcal} / \mathrm{mol}$ (favorable)

$\Delta S=-21.5 \mathrm{Cal} /$ grado * mol reacción (desfavorable)

$$
\mathrm{E}^{0}=+3.98 \mathrm{~V}
$$

¿De dónde proviene esta alta favorabilidad del cambio entálpico? (Los efectos de red o ambientales son cruciales). 


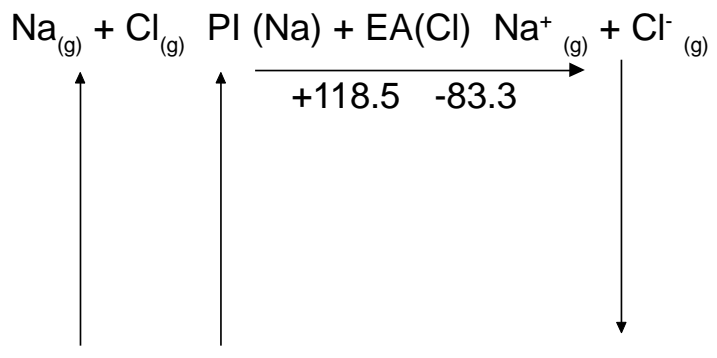

$\Delta \mathrm{H}_{\text {sub }}+25.6 \mathrm{ED}+28.6 \quad \Delta \mathrm{H}_{\text {crist }}-187.6$

$\mathrm{Na}{ }_{(\mathrm{s})}+1 / 2 \mathrm{Cl}_{2(\mathrm{~g})} \quad \Delta \mathrm{H}_{\text {reac }}{ }^{\circ} \quad \mathrm{NaCl}_{(\mathrm{s})}$

$-98.2$

La parte superior de este ciclo representa las cualidades intrínsecas de atracción de electrones del átomo de cloro y el catión sodio, energéticamente desfavorable.

Sólo la tremenda diferencia en estabilidad de la red, entre el $\mathrm{NaCl}$ iónico y el menos fuerte enlazamiento de $\mathrm{Na}^{0}$ y $\mathrm{Cl}_{2}$ hace que la reacción sea favorable.

Algunas reacciones de esfera exterior no muestran contribuciones ambientales para todo el cambio entálpico:

$$
\begin{aligned}
& \mathrm{Fe}(\mathrm{CN})_{6}^{-4}{ }_{(\mathrm{ac})}+\mathrm{Mo}(\mathrm{CN})_{8}^{-3}{ }_{(\mathrm{ac})} \longrightarrow \mathrm{Fe}(\mathrm{CN})_{6}^{-3}(\mathrm{ac}) \\
& \Delta \mathrm{G}^{0}=8.5 \mathrm{Kcal} / \mathrm{mol}(\mathrm{CN})_{8}^{-4}(\mathrm{ac}) \\
& \mathrm{E}^{\mathrm{o}}=0.37 \mathrm{~V}
\end{aligned}
$$

Para mecanismos de reacciones de esfera interior, la fuente de energía es diferente.

Estos mecanismos van acompañados por transferencia de átomos:

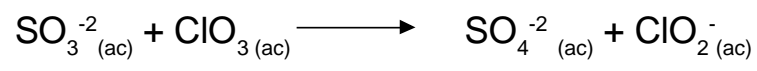

$\Delta \mathrm{G}^{0}=-53.6 \mathrm{Kcal} / \mathrm{mol}$ (espontanea)

$\Delta \mathrm{H}^{0}=-59.9 \mathrm{~K} / \mathrm{cal} / \mathrm{mol}$ (favorable)

$\Delta S=-21.1 \mathrm{Cal} /$ grado * mol reacción (desfavorable)

$$
E^{0}=+1.16 \mathrm{~V}
$$


Efectos ambientales-energías solvatación son menos importantes que la diferencia en energías de enlace para el átomo que se ha transferido.

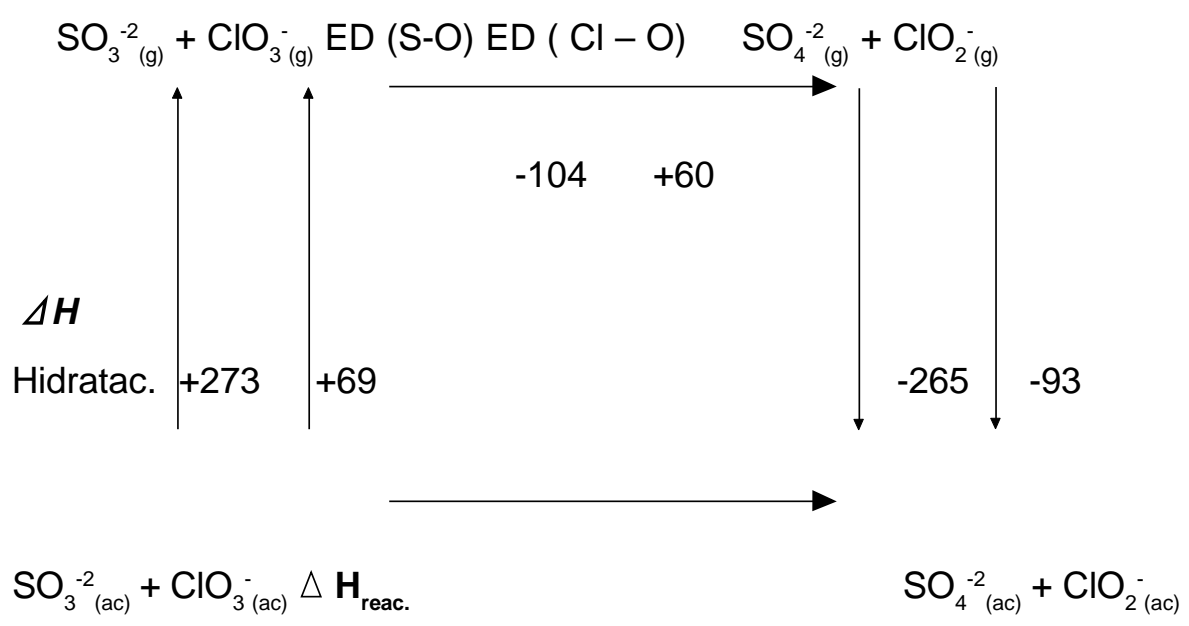

Las energías de redes cristalinas no se pueden medir directamente. Sin embargo, aplicando la Ley de Hess a una serie de reacciones que se inician con la combinación del Sodio $\left(\mathrm{Na}{ }_{(\mathrm{s})}\right)$ y el Cloro $\left(\mathrm{Cl}_{2(\mathrm{~g})}\right)$ y terminan con la formación de Cloruro de Sodio ( $\mathrm{NaCl}$ ) cristalino

Tal secuencia de reacciones para sustancias iónicas se llama Ciclo de BornHaber.

¿Cuál es el calor de reacción para el siguiente sistema?

$$
\mathrm{Na}{ }_{(\mathrm{s})}+1 / 2 \mathrm{Cl}_{2(\mathrm{~g})} \longrightarrow \mathrm{NaCl}_{(\mathrm{s})} \quad \Delta \mathrm{H}_{\text {reac }}=-98.2 \mathrm{kCal}
$$

Las siguientes reacciones y datos experimentales pueden sumarse para obtener los mismos resultados:

1. Sublimación de sodio metálico (vaporización). El cambio calórico es $\Delta \mathrm{H}_{\mathrm{f}}$, que para

$\mathrm{Na}_{(\mathrm{g})(\text { metálico) }}=\Delta \mathrm{H}_{\text {subl. }}$

$\mathrm{Na}_{(\mathrm{s})} \longrightarrow \mathrm{Na}_{(\mathrm{g})} \quad \Delta \mathrm{H}_{\text {subl }}=26.0 \mathrm{Kcal}$ 
2. Disociación $1 / 2 \mathrm{Cl}_{2(\mathrm{~g})}$ en átomos de $\mathrm{Cl}_{2(\mathrm{~g})}$. El cambio calórico es? $\Delta \mathrm{H}_{\mathrm{F}}$. Para los átomos de cloro gaseoso, $\mathrm{Cl}_{(\mathrm{g})}=1 / 2 \Delta \mathrm{H}$

$1 / 2 \mathrm{Cl}_{2(\mathrm{~g})}$ disociación $\longrightarrow \mathrm{Cl}_{(\mathrm{g})} \quad \Delta \mathrm{H}_{\text {dis }}=29.1 \mathrm{Kcal}$

3. Ionización de $1 \mathrm{~mol}$ de átomos de sodio $(\mathrm{Na})$. El cambio calórico es la energía de disociación para sodio $(\mathrm{Na})$.

$\mathrm{Na}$

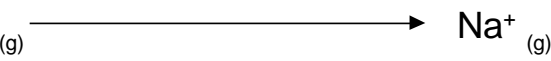

$\Delta \mathrm{H}_{\mathrm{El}}=118 \mathrm{Kcal}$

4. Adición de $1 \mathrm{~mol}$ de electrones a $1 \mathrm{~mol}$ átomos de cloro gaseoso $\mathrm{Cl}_{(\mathrm{g})}$. Este cambio calórico es afinidad electrónica del cloro.

$\mathrm{Cl}_{(\mathrm{g})}+\mathrm{e}^{-} \longrightarrow \mathrm{Cl}^{-}{ }_{(\mathrm{g})} \quad \Delta \mathrm{H}_{\mathrm{EA}}=-83 \mathrm{Kcal}$

5. Condensación de los iones gaseosos para formar $1 \mathrm{~mol}$ de $\mathrm{NaCl}_{(\mathrm{s})}$. Este cambio calórico no se puede medir directamente.

$\mathrm{Na}^{+}{ }_{(\mathrm{g})}+\mathrm{Cl}^{-} \longrightarrow \mathrm{NaCl}_{(\mathrm{g})} \quad \Delta \mathrm{H}_{\text {cristal }}=?$

La suma de éstas cinco reacciones y los valores de ? $\mathrm{H}$, nos permiten calcular la $\Delta H$ cristal.

1. $\mathrm{Na}_{(\mathrm{s})}$ $\mathrm{Na}$

$\Delta \mathrm{H}_{\text {subl }}=26.0 \mathrm{Kcal}$

2. $1 / 2 \mathrm{Cl}_{2(\mathrm{~g})}$

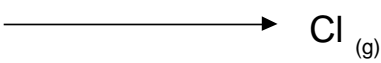

$1 / 2 \Delta H_{\text {dis }}=29.1 \mathrm{Kcal}$

3. $\mathrm{Na}$

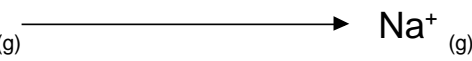

$\Delta \mathrm{H}_{\mathrm{El}}=118 \mathrm{Kcal}$

4. $\mathrm{Cl}_{(\mathrm{g})}+\mathrm{e}$<smiles>Cl[Hg]Cl</smiles>

$\Delta H_{E A}=-83 \mathrm{Kcal}$

5. $\mathrm{Na}^{+}{ }_{(\mathrm{g})}+\mathrm{Cl}$

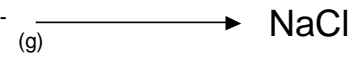

(s)

$\Delta \mathrm{H}_{\text {cristal }}=$ ?

$\mathrm{Na}{ }_{(\mathrm{s})}+1 / 2 \mathrm{Cl}_{2(\mathrm{~g})}$

$\mathrm{NaCl}_{(\mathrm{s})}+\Delta \mathrm{H}_{\text {cristal }}=?$ 
$\Delta \mathrm{H}_{\text {reacción }}=\Delta \mathrm{H}_{\text {Sub }}+\Delta \frac{1}{2} \Delta \mathrm{H}_{\text {diso }}+\Delta \mathrm{H}_{\mathrm{El}}+\Delta \mathrm{H}_{\mathrm{EA}}+\Delta \mathrm{H}_{\text {cristal }}=?$

$-98.2 \mathrm{Kcal}=26.0 \mathrm{Kcal}+29.1 \mathrm{Kcal}+118 \mathrm{Kcal}+(-83 \mathrm{Kcal})+\Delta \mathrm{H}_{\text {cristal }}$

$\mathrm{H}_{\text {cristal }}=\Delta \mathrm{H}_{\text {cristal }}=-188.1 \mathrm{Kcal} / \mathrm{mol} \mathrm{NaCl}{ }_{(\mathrm{s})}$

¿Qué relaciones interconceptuales se dan entre los conceptos oxidación, reducción, transferencia de electrones, acidos, bases y combustión? ¿Cuales son las implicaciones científicas, tecnológicas, sociales y ambientales de estas relaciones?

\section{Combustión}

La combustión es una reacción de oxidación- reducción de un comburente y un combustible, acompañada de cambios de energía.

¿Qué relaciones existen entre los conceptos fuego, combustible, combustión y oxidación?

\section{Gas natural}

Es una mezcla de metano $\left(\mathrm{CH}_{4(\mathrm{~g})}\right)(85 \%)$, etano $\left(\mathrm{C}_{2} \mathrm{H}_{6}\right)(10 \%)$, propano $\left(\mathrm{C}_{3} \mathrm{H}_{8}\right)$, butano $\left(\mathrm{C}_{4} \mathrm{H}_{10}\right)$ y nitrógeno $\left(\mathrm{N}_{2}\right)(5 \%)$. La combustión de hidrocarburos con oxidación completa son procesos químicos ideales que se presentan raras veces en condiciones experimentales y tecnológicas especiales. Durante los procesos de oxidación incompleta, que son los más frecuentes, las temperaturas son muy bajas y la cantidad de oxígeno suministrado (comburente) no es suficiente para que las moléculas del combustible alcancen el estado más alto de oxidación. En este caso sus moléculas escapan parcialmente oxidadas o en estado original. La combustión completa del metano originaría los siguientes productos:

$$
\mathrm{CH}_{4(\mathrm{~g})}+2 \mathrm{O}_{2(\mathrm{~g})}+\stackrel{\text { energía activación }}{\longrightarrow} \mathrm{CO}_{2(\mathrm{~g})}+2 \mathrm{H}_{2} \mathrm{O}_{(\mathrm{g})}-\mathrm{E}
$$

donde E equivale a la entalpía de combustión a 500 K, y es igual a -800.58 $\mathrm{kj} / \mathrm{mol}$

La combustión incompleta del metano origina los siguientes productos:

$$
\mathrm{CH}_{4(\mathrm{~g})}+\mathrm{O}_{2(\mathrm{~g})}+\stackrel{\text { energía activación }}{\longrightarrow} \mathrm{CO}_{2(\mathrm{~g})}+\mathrm{H}_{2} \mathrm{O}_{(\mathrm{g})}+\mathrm{E}+\mathrm{CO}_{(\mathrm{g})}+\mathrm{C}_{(\text {hollin) }}+\mathrm{CH}_{4(\mathrm{~g})}
$$


El rendimiento energético de esta reacción es inferior y las especies contaminantes son mayores.

En el caso de la combustión incompleta del gas natural con todos sus constituyentes, la concentración de las especies contaminantes se incrementaría $\mathrm{y}$, por consiguiente, los riesgos.

La segunda ecuación no corresponde a una reacción estequiométrica, por cuanto se forman diferentes productos en diferentes proporciones cada vez que tenga lugar la combustión. Ninguna ecuación sencilla balanceada puede representar las diferentes reacciones que suceden.

\section{Emisiones de $\mathrm{CO}_{2}$ a la atmósfera}

Los gases invernadero $\mathrm{CO}_{2}, \mathrm{CH}_{4} \mathrm{~N}_{2} \mathrm{O}, \mathrm{CFC}$ (dióxido de carbono, metano, monóxido de dinitrógeno y clorofluorocarbonados), cuyas concentraciones en la troposfera terrestre son pequeñas, permiten el paso de la radiación visible hacia la Tierra, la cual es absorbida, reflejada y devuelta al espacio en forma de radiación infrarroja que es atrapada por gases invernadero. Este proceso permite que la temperatura promedio sobre la superficie terrestre sea aproximadamente $15^{\circ} \mathrm{C}$. Se ha estimado que la combustión de combustibles fósiles y no fósiles está aportando grandes cantidades de $\mathrm{CO}_{2}$ a la atmósfera y contribuyendo al $50 \%$ del calentamiento global (Kaufman 2000) al retener (con otros gases) la radiación infrarroja devuelta por la superficie terrestre.

\section{Dureza temporal y total del agua}

El calcio y magnesio, y algunas veces el ión ferroso $\mathrm{Fe}^{++}$, son los responsables de la dureza total y temporal del agua. La presencia de iones Calcio $\mathrm{Ca}^{++}$y bicarbonato $\mathrm{HCO}_{3}^{-}$(provenientes de minerales) son los causantes de la dureza temporal del agua, que puede eliminarse por ebullición (Manaham, 1994).

$$
\mathrm{Ca}^{++}{ }_{(\mathrm{ac})}+2 \mathrm{HCO}_{3(\mathrm{ac})}^{-} \rightleftarrows \mathrm{CaCO}_{3(\mathrm{~s})}+\mathrm{CO}_{2(\mathrm{~g})}+\mathrm{H}_{2} \mathrm{O}
$$

La ecuación anterior representa la solubilidad del carbonato de calcio en agua en presencia de $\mathrm{CO}_{2}$. Un aumento de temperatura remueve el $\mathrm{CO}_{2}$ y la dureza temporal del agua, formando carbonato de calcio, $\mathrm{CaCO}_{3}$, por desplazamiento de la reacción hacia la derecha.

En el agua, carbonatos y bicarbonatos actúan como aceptores de protones y como amortiguadores del $\mathrm{pH}$ (buffers). 
Fotosíntesis de la biomasa como fuente de carbohidratos y alternativa de energía renovable

El $\mathrm{CO}_{2}$ atmosférico es clave para la síntesis de la biomasa (biomoléculas)

$$
\mathrm{CO}_{2(\mathrm{~g})}+\mathrm{H}_{2} \mathrm{O}_{(\mathrm{l})}+\longrightarrow\left(\mathrm{CH}_{2} \mathrm{O}\right)+\mathrm{O}_{2(\mathrm{~g})}
$$

El $\mathrm{CO}_{2}$ y el $\mathrm{HCO}_{3}^{-}$en el agua, contribuyen a formar biomasa (fotosíntesis acuática)

$$
\begin{aligned}
& \mathrm{CO}_{2}+\mathrm{H}_{2} \mathrm{O}_{(\mathrm{l})} \stackrel{\text { Fotones }}{\longrightarrow} \mathrm{CH}_{2} \mathrm{O}+\mathrm{O}_{2} \\
& \mathrm{HCO}_{3}+\mathrm{H}_{2} \mathrm{O} \underset{(\mathrm{l})}{\stackrel{\text { Fotones }}{\longrightarrow} \mathrm{CH}_{2} \mathrm{O}+\mathrm{O}_{2}+\mathrm{OH}^{-}+\mathrm{H}_{2} \mathrm{O}}
\end{aligned}
$$

En aguas subterráneas la degradación de la biomasa aporta $\mathrm{CO}_{2}$ de gran importancia para facilitar la solubilidad de $\mathrm{CaCO}_{3}$, el cual libera $\mathrm{Ca}^{++}$ (Manaham, 1994).

Reacciones anaeróbicas de oxidación-reducción de biomasa en agua con enzimas como catalizadores (Manaham, 1994)

$$
\begin{array}{ll}
1 / 4 \mathrm{CH}_{2} \mathrm{O}+1 / 4 \mathrm{H}_{2} \mathrm{O} \stackrel{\text { Enzimas }}{\longrightarrow} 1 / 4 \mathrm{CO}_{2(\mathrm{~g})}+\mathrm{H}^{+}+\mathrm{e}^{-} \\
\stackrel{1 / 8 \mathrm{CO}_{2(\mathrm{~g})}+\mathrm{H}^{+}+\mathrm{e}^{-} \longrightarrow \text { Enzimas }}{\longrightarrow} 1 / 8 \mathrm{CH}_{4(\mathrm{~g})}+1 / 4 \mathrm{H}_{2} \mathrm{O} \\
\hline 1 / 4 \mathrm{CH}_{2} \mathrm{O} \longrightarrow 1 / 8 \mathrm{CH}_{4(\mathrm{~g})}+1 / 8 \mathrm{CO}_{2(\mathrm{~g})}
\end{array}
$$


¿Qué implicaciones científicas, tecnológicas y sociales representan las diversas interacciones químicas de los productos de combustión del gas natural y de otros combustibles fósiles en el ambiente?

\section{EXPERIMENTACIÓN}

El planeamiento de actividades de experimentación tendrá en cuenta diversas clases de combustibles que sean de uso frecuente para la generación de energía, Por ejemplo: combustión de gas propano, combustión de hulla, combustión de madera y combustión de gas natural.

\section{Probiema de Investigación}

Para articular el trabajo de experimentación con los problemas de investigación, se plantean las siguientes preguntas que orientan el proceso de investigación:

1. ¿Cuál es la disponibilidad y demanda de los combustibles mencionados en un sector específico?

2. ¿Cuál es la frecuencia de uso y el costo de cada combustible?

3. ¿Cuál es la eficiencia energética de cada combustible?

4. ¿Qué clase de productos genera cada combustión?

5. ¿Qué alteraciones ambientales ocasionan dichos productos y cuál es su incidencia en la salud humana y ambiental?
6. ¿Qué alternativas energéticas renovables se proponen para mejorar la calidad de vida?

\section{STUACIONES DE RIESGO} Generadas

La combustión que utiliza gas natural, leña, hulla, gas propano, metanol, gasolina y otros combustibles genera riesgos de diversa índole. Las emisiones gaseosas modifican las concentraciones de los gases invernadero y de los gases traza, ocasionando un desequilibrio calórico y una serie de reacciones fotoquímicas que producen problemas como lluvia ácida, lluvia seca y smog fotoquímico, los cuales tienen profundas implicaciones en el equilibrio de los ecosistemas y en la sostenibilidad ambiental.

\section{Estrategias de Mango}

Educativas. Comprender como inciden las actividades y los fenómenos de la cotidianidad en la generación de problemas ambientales y cómo pueden ser abordados desde el aula para su investigación y manejo.

Tecnológicas. Utilizar de tecnologías que permitan la generación de energía renovable a partir de residuos orgánicos, carbohidratos, lípidos y estiércol, y contribuyan a mejorar la calidad ambiental.

Ético-legal. La estructuración de conocimiento debe orientar a las personas en la reflexión sobre las implicaciones legales, 
éticas, económicas y sociales relacionadas con su actividad en el contexto de la sostenibilipersonal, familiar y comunitaria dad ambiental.

\section{Competencias}

Las competencias se establecerán de acuerdo con la intención de la estrategia, para propiciar el desarrollo de la capacidad de reflexionar, argumentar y proponer, sobre relaciones interconceptuales, las transformaciones químicas en el ambiente, su incidencia con situaciones riesgosas y las implicaciones científicas, tecnológicas, sociales y ambientales de las actividades humanas.

En el transcurso del trabajo experimental, se espera apoyar el desarro- llo de destrezas y habilidades para manipular instrumentos, obtener información confiable, graficar e interpretar el significado de los datos y los resultados desde una visión compleja del ambiente.

Con el conocimiento construido sobre la incidencia de las actividades químicas en el ambiente, se espera identificar situaciones ambientales riesgosas que puedan constituir problemas objeto de investigación y estrategias de manejo.

\section{Conclusiones}

- Las actividades humanas y los procesos químicos pueden ser formulados como objetos de trabajo académico desde la perspectiva conceptual, experimental, investigativa y ambiental.

- El desarrollo de estas actividades o procesos químicos en el aula, mediante conceptos integradores y núcleos problemáticos, permite al estudiante establecer las implicaciones de estas actividades con la ciencia, la tecnología, la sociedad y el ambiente.

- Una actividad humana o proceso químico puede servir como referente de un problema de investigación en un contexto interdisciplinar y multidisciplinar.

Desde esta perspectiva integradora, el conocimiento construido por el individuo debe generar un cambio de comportamiento que le permita asumir mayor responsabilidad y compromiso para manejar y resolver tales situaciones.

Este enfoque en enseñanza de la química, amplía las oportunidades para que en el desarrollo de las actividades académicas se articulen con las relaciones ciencia, tecnología, sociedad y ambiente. 


\section{Bibliografía}

Guerrero, J. y Cabrera L. (2002). Enseñanza de las Ciencias Experimentales, mediante enfoque interdisciplinar utilizando el Impacto ambiental generado por emisiones de $\mathrm{CO}_{2}$ Revista Tecné, Episteme y Didaxis, 12. Facultad de Ciencia y Tecnología, UPN. Bogotá D.C.

Guerrero, J. (2000). Impacto ambiental generado las actividades químicas de producción y uso de fluorocarbonos. Revista Tecné, Episteme y Didaxis, 7. Facultad de Ciencia y Tecnología UPN. Bogotá D.C.

Guerrero, J. (2003). Actividades humanas y enseñanza de la química en el contexto ambiental. Revista Tecné, Episteme y Didaxis. número extraordinario.

Newton, D. (1991). Environmental Chemistry. Portlan, Maine: Weston Walch Publisher.

Primer Congreso de Formación de Profesores en Ciencias. Universidad Pedagógica Nacional. Bogotá Colombia.

The Regency Corporation Limited en colaboración el PNUMA (1998). Una empresa con futuro. El desarrollo económico y la tecnologías ecológicas. 